\title{
La ville de Québec comme foyer « libéral » au XIXe siècle
}

Les catholiques canadiens-français entre opportunisme et ultramontanisme

The city of Quebec as a "liberal" center in the $19^{\text {th }}$ century. French Canadian Catholics between opportunism and ultramontanism

La ciudad de Quebec como foco "liberal" en el siglo XIX. Los católicos francocanadienses entre oportunismo y ultramontanismo

\section{Jean-Philippe Warren}

\section{(2) OpenEdition}

\section{Journals}

Édition électronique

URL : http://journals.openedition.org/assr/25831

DOI : $10.4000 /$ assr.25831

ISSN : $1777-5825$

\section{Éditeur}

Éditions de l'EHESS

Édition imprimée

Date de publication : 2 mars 2014

Pagination : 227-244

ISBN : 978-2-7132-2431-7

ISSN : 0335-5985

\section{Référence électronique}

Jean-Philippe Warren, «La ville de Québec comme foyer « libéral » au XIXe siècle », Archives de sciences sociales des religions [En ligne], 165 । janvier-mars 2014, mis en ligne le 20 février 2017, consulté le 03 mai 2019. URL : http://journals.openedition.org/assr/25831; DOI : 10.4000/assr.25831 


\title{
Jean-Philippe Warren
}

\section{La ville de Québec comme foyer "libéral " au XIXe siècle}

\author{
Les catholiques canadiens-français \\ entre opportunisme et ultramontanisme ${ }^{1}$
}

Je n'aurais pas osé le dire, mais c'est écrit dans un document publié partout : Québec et Montréal sont deux villes ennemies. Ce n'est que trop vrai! Quel triste destin! Deux villes qui ont toutes les raisons du monde de s'aimer et qui se détestent, rivales partout, amies nulle part ou bien rarement. (A. Duclos de Celles, $1881: 416$ )

La rivalité entre les villes de Québec et de Montréal remonte à très loin, si loin qu'elle fait presque partie du folklore ${ }^{2}$. On peut toutefois affirmer que cette opposition entre les deux plus grandes agglomérations de la province de Québec a pris une tournure pour ainsi dire dramatique à la fin du XIX siècle avec l'irruption sur la scène publique d'une myriade d'affaires qui déchaînèrent les passions des Canadiens de langue française.

En un clin d'œil, prétendait le journaliste Alfred Duclos De Celles en septembre 1881, les esprits sont montés si haut qu'ils pouvaient monter. Polémiques et discussions ont fait rage, et tout a été chauffé à blanc. Jamais, dans les luttes soit contre nos amis d'Ontario, soit dans les disputes avec les journaux protestants, on n'a fait pareilles dépenses d'acrimonie et de fiel. [...] C'était la guerre civile, quoi, et il soufflait de toutes parts un vent fratricide. (A. Duclos de Celles, $1881: 416$ )

Bien que cette déclaration fût un brin exagérée, elle mettait le doigt sur des tensions réelles qui menaçaient de compromettre la bonne marche des événements dans la province. Dans la deuxième moitié du XIX siècle, l'antagonisme

1. Je remercie Florian Michel, Guy Laperrière et Christian Blais pour leurs critiques et commentaires sur une version préliminaire de ce texte.

2. "La rivalité Québec-Montréal n'était pas un phénomène nouveau au début du XIX ${ }^{\mathrm{e}}$ siècle. Elle datait du XVII ${ }^{\mathrm{e}}$ siècle » (F. Ouellet, 1959 : 312). Parlant du XVII ${ }^{\mathrm{e}}$ siècle, Télesphore Saint-Pierre écrivait : "La rivalité entre Québec et Montréal se manifestait toujours à chaque occasion " (T. Saint-Pierre, 1894 : 9). Notons que les couleurs politiques locales des deux agglomérations trouveront une manifestation au $\mathrm{XX}^{\mathrm{e}}$ siècle dans des Écoles historiographiques et littéraires rivales (A. Hayward, 1991 : 514-524 ; R. Rudin, 1997). Pour une interprétation globale, lire P. Villeneuve (1981). 
entre Québec et Montréal s'est très souvent manifesté lors de polémiques religieuses $^{3}$. Les disputes théologiques québécoises suivaient, en quelque sorte, des fractures géographiques. Or, contrairement à ce que l'on pourrait penser, ce n'est pas Montréal (qui s'imposait à ce moment comme la métropole industrielle et démographique du Canada) qui incarnait à cette époque, aux yeux de plusieurs observateurs, le haut lieu de la tolérance religieuse, mais plutôt Québec. Arthur Buies, par exemple, ancienne chemise rouge de Garibaldi aux penchants anticléricaux, avait décidé à son retour d'Italie d'élire pendant un certain temps domicile dans la capitale, car il espérait profiter du fait que le clergé y reconnaissait au moins, contrairement à celui de la métropole, la légitimité du libéralisme de type britannique (M. Gagnon, 1965). Des prêtres conservateurs de Montréal et Trois-Rivières, les deux principaux foyers de l'ultramontanisme dans la province, ne se gênaient pas pour dénoncer publiquement cette réceptivité plus grande à la libre-pensée.

«Babylone, disait l'un d'entre eux, c'est la division, la confusion, le désordre; en un mot, l'enfer. Plaçons Babylone. Supposons que Babylone soit Québec. Montréal représentera Jérusalem » ${ }^{4}$.

La question dès lors se pose : comment se fait-il que le catholicisme canadienfrançais a semblé se montrer plus ouvert dans une ville que dans l'autre ? Qu'estce qui explique ces différences d'attitudes et d'idées? Si une série de facteurs à la fois contingents et structuraux peuvent sans doute éclairer ces divergences entre Québec et Montréal, nous nous attarderons ici sur un élément qui paraît avoir influencé, entre autres, la structuration des rapports entre Église catholique et pouvoir séculier au Québec au XIXe siècle, à savoir les dynamiques sociopolitiques propres à la capitale québécoise, dynamiques liées à la plus ou moins grande proximité des élites religieuses avec le pouvoir civil. En revenant sur ce facteur, ce texte offre une première lunette d'approche pour saisir la spécificité de Québec comme capitale d'une colonie, puis d'une province, avec ce que cela suppose d'effet grossissant. Parce qu'elle porte sur un contexte où les catholiques canadiens-français sont doublement minoritaires par la foi et la langue, cette recherche soulève aussi un questionnement original sur les relations entre les réalités propres au siège du gouvernement et les stratégies de développement et de rayonnement de l'Église catholique.

3. Sur le catholicisme québécois durant la période étudiée, lire P. Sylvain et N. Voisine (1991). Sur les idées au Québec, lire Y. Lamonde (2000). Sur les relations entre pouvoir civils et religieux, lire L. Ferguson (2009). Sur l'histoire des relations entre Rome et le Canada, lire R. Perin (1993).

4. Sermon du père Ponche à Trois-Rivières, 1883, ASTR, cité par A. Lavallée (1974 : 164). 


\section{Le conte de deux cités}

Mandaté par le Vatican pour enquêter sur les débats qui agitaient le clergé canadien en 1875, le cardinal Camillo Santori avait conclu que les dissidences entre Montréal et Québec étaient aggravées par «les rapports peu amicaux qu'entretiennent malheureusement entre eux leurs clergés respectifs » (R. Perin, 2008 : 249). Les inimitiés personnelles, théologiques et idéologiques entre les membres de la haute hiérarchie épiscopale prenaient spontanément la tournure d'une guerre entre les principales villes provinciales. D'un côté, il y avait Ignace Bourget (1799-1885), évêque de Montréal (1840-1876), et Louis-François Laflèche (1818-1898), évêque de Trois-Rivières (1870-1898), deux hommes qui avaient assisté à Rome, en juillet 1870, à la proclamation de l'infaillibilité papale et qui incarnaient dans la province l'orthodoxie ultramontaine avec le plus de superbe. De l'autre côté, il y avait Elzéar-Alexandre Taschereau (1820-1898), nommé évêque de Québec en 1871, puis premier cardinal canadien en 1886, qui venait d'une très éminente famille de notables libéraux de la ville de Québec ${ }^{5}$. Wilfrid Laurier, le futur premier ministre du Canada (et député de Québec-Est pendant 41 ans), fit lui-même l'éloge de la famille Taschereau en 1892 (discours cité P.-G. Roy, 1901 : 7-8).

Refusant tout compromis avec le libéralisme catholique ou le libéralisme politique de type républicain, Mgr Taschereau s'entendait pour tolérer le libéralisme de type britannique, c'est-à-dire un libéralisme non révolutionnaire et non anticlérical. Ce distinguo ne semblait pas suffisant pour la frange la plus conservatrice de l'Église canadienne-française. Taschereau eut par conséquent maille à partir avec les membres les plus rigides du clergé québécois, lesquels suspectaient des conspirations maçonniques partout ; pour eux, le simple mot de libéralisme représentait une véritable insulte sans avoir besoin de plus de définition. Déjà, alors qu'il dirigeait le séminaire de Québec, le futur cardinal avait suscité de farouches réticences face à son enseignement jugé trop conciliant. Même après son ordination épiscopale, les murmures contre sa gouverne ne cessèrent point, plusieurs prêtres dénonçant le fait qu'il aurait manœuvré pour muter à des postes peu avantageux ceux qui n'affichaient pas assez de zèle à défendre ses opinions et, inversement, pour récompenser ceux qui prenaient soin de s'éloigner d'un ultramontanisme trop "ultramonté » (selon une expression péjorative courante à l'époque). Dans une brochure publiée en 1873, Il y a du libéralisme et $d u$ gallicanisme en Canada, un auteur anonyme se plaignait de ce trafic d'influence.

5. Par exemple, son frère Jean-Thomas, fut juge de la Cour supérieure de Québec (18551875), juge de la Cour du Banc de la Reine (1873-1875) et magistrat de la Cour suprême du Canada (1875-1878). Un de ses neveux, Henri-Thomas, fut député de Montmagny (18721878), juge de la Cour supérieure de Québec et juge en chef de la Cour du Banc du Roi. Quant à sa mère, elle était la nièce de Mgr Bernard-Claude Panet, le successeur de Mgr Octave Plessis sur le siège épiscopal de Québec. 
Qui aurait pu imaginer, s'indignait-il, que la nomination aux cures et à n'importe quel office ou bénéfice ecclésiastique deviendrait ainsi, contrairement à toutes les prescriptions du droit canonique, un moyen d'exercer une espèce d'apostolat en faveur des idées gallicanes et libérales ${ }^{6}$ ? (Luigi [Alexis Pelletier], $1873: 31$ )

Les différends entre Québec et Montréal ne furent sans doute jamais mieux illustrés que lors de la querelle universitaire (1876-1891). En bref, Montréal voulait qu'on lui concède une université autonome et l'Université Laval, à Québec, ne voulait rien céder de son monopole. Ce conflit reposait certes en grande partie sur des considérations monétaires, mais il avait aussi des bases idéologiques : comme Québec représentait dans la province le "clan libéral », plusieurs catholiques souhaitaient soustraire l'enseignement à son emprise. Bourget regrettait le premier que l'Université Laval soit " entachée de gallicanisme » et en tirait la conclusion qu'il fallait se presser de fonder à Montréal un établissement universitaire inspiré par des principes plus orthodoxes. L'Université de Montréal qu'il appelait de ses vœux devait être, disait-il, " assez ultramontaine pour tenir en bride celle de Laval » (Bourget à Joseph Desautels, le 2 août 1872, cité par P. Desjardins, 1944 : 284). En retour, les esprits libéraux favorisaient l'Université Laval dans ses démêlés avec Montréal parce qu'ils trouvaient en elle "l'institution la plus libérale que nous ayons, et parce que le groupe ultramontain [...] dont Mgr Bourget a besoin, est opposé à l'université » ([s.a.], 1881a : 4).

Ce conflit autour de la fondation d'une université autonome à Montréal a mis en présence les deux évêchés de Montréal et Québec, les sympathisants du Parti libéral et du Parti conservateur, les partisans d'une interprétation souple ou rigide de la doctrine catholique et les élites citoyennes des deux principales villes de la province. Tout au long de la querelle universitaire, comme le résumait l'historien Robert Rumilly, "les rivalités de personnes, de partis, d'institutions et même de villes » se sont greffées "sur le conflit d'idées générales » (R. Rumilly, 1962 : 99 ; lire aussi M. Rheault, 2011). À la fin, l'on peut dire que l'antagonisme était autant celui entre Bourget Taschereau, celui entre les libéraux et les conservateurs et celui entre les catholiques non intransigeants et les ultramontains, que celui entre les villes de Québec et Montréal elles-mêmes. Ces tensions urbanocentristes illustrent tout autant de banales guerres de clochers entre deux cités qui rivalisent pour la domination du champ provincial que de réelles oppositions religieuses et politiques marquées par des cultures urbaines historiquement distinctes. De 1867 (année de la signature de l'acte de l'Amérique du nord britannique) à 1892 (date de la refonte de la carte électorale), les circonscriptions de Québec (Centre, Est et Ouest) ont envoyé trois fois plus de députés libéraux que de députés conservateurs à Ottawa, alors que celles de Montréal (Centre, Est et Ouest, ainsi que Hochelaga et Jacques-Cartier) ont envoyé près de deux fois plus de députés conservateurs que de députés libéraux. Quant aux électeurs de

6. Sur l'ensemble de ces débats en lien avec le libéralisme de Taschereau, lire J.-C. Dupuis (2006). 
Trois-Rivières, ils réélisaient sans interruption pendant cette période des candidats conservateurs.

L'opposition entre Montréal et Trois-Rivières, d'une part, et Québec d'autre part, ne signifie pas que la pensée libérale ne comptait pas d'adeptes dans la métropole ou que les partisans de la pensée ultramontaine aient été absents de la capitale. Au contraire, si l'ultramontanisme a été plus vigoureux au sein du clergé de Montréal, cela tient en partie à la présence de forces progressistes plus remuantes et plus puissantes dans la société civile - dont l'Institut canadien, un lieu de rendez-vous des "Rouges " ${ }^{7}$, ou les journaux L'Avenir (1847-1857), Le Pays (1852-1871) et La Patrie (1879-1896). Réciproquement, Québec ne manquait pas à l'époque de farouches défenseurs de l'orthodoxie - dont le journaliste Jules-Paul Tardivel, rédacteur de l'hebdomadaire la Vérité (1881-1923), et les membres ultramontains du Cercle catholique (1876-1897). Mais ces gens étaient en minorité au sein du clergé de la capitale. Louis-Nazaire Bégin, futur cardinal de Québec, trahissait l'opinion de maints autres prêtres de son évêché quand il écrivait dans une lettre privée : «Ce Tardivel est un être venimeux, on dirait qu'il s'est donné la mission de souiller tout ce qu'il touche " (cité par M. Girard, 1967 : 398). Les membres du cercle ultramontain eurent eux aussi à subir les critiques de Taschereau (P. Savard, 1967).

Les commentaires persifleurs, les tractations sourdes et les querelles ouvertes entre Québec et Montréal étaient devenus monnaie courante dans la deuxième moitié du XIX siècle. Pour régler ces disputes, on n'hésitait pas à faire parvenir au Vatican des lettres, des rapports, des pétitions, quand on n’y déléguait pas tout simplement des représentants ou des ambassades. Les bureaux du SaintSiège étaient inondés de suppliques et d'entrevues. On prétendait que la province de Québec envoyait à Rome plus de correspondance et de délégations que l'ensemble de la chrétienté réunie. À l'évidence, ces querelles ne reposaient pas exclusivement sur les personnalités fortes des acteurs en présence, même si cellesci pesaient d'un poids considérable sur les événements diocésains. Il serait certes risible de vouloir expliquer le libéralisme du clergé de Nicolet ou de Valleyfield par le milieu social, mais il serait tout aussi aveugle de nier entièrement les subtils positionnements politiques provoqués par le compagnonnage des gens d'influence. Les décisions d'un Taschereau (qui appartenait par sa famille, son éducation et ses amis au réseau de la ville de Québec) reflétaient les intérêts de sa localité aussi bien que ses penchants propres - quoiqu'à des degrés inégaux. De même, Bourget et Laflèche exprimaient par leur conduite des affaires de leur évêché les vues de leur milieu. Le libéralisme et le gallicanisme supposés de l'un, et le conservatisme et l'ultramontanisme des autres s'alimentaient en partie aux sources de cultures urbaines spécifiques.

7. Héritier du défunt Parti patriote, le Parti rouge était formé de radicaux et de réformistes libéraux et républicains. Opposés aux idées ultramontaines du clergé, ses membres les plus modérés allaient former le Parti libéral du Canada (J.-P. Bernard, 1971). 


\section{L'opportunisme de Québec}

Aux yeux de la classe politique de Montréal, l'élite québécoise a toujours paru suspecte d'opportunisme, les habitants de la capitale ayant tendance à adopter historiquement des opinions plus prudentes et temporisatrices face au pouvoir politique dominant (F. Ouellet, 1959 : 311 ; P. Sylvain et N. Voisine, 1991 : 240). C'est ainsi que Québec a semblé une cité plus loyaliste pendant une bonne partie du XIX siècle. On sait que lors de la Rébellion de 1837, les principaux leaders nationalistes de la capitale ont refusé de suivre la faction la plus radicale du Parti patriote et ont prêché la soumission aux puissances établies (A. Greer et L. Robichaud, 1989). Ce respect du pouvoir colonial ne s'est pas démenti par la suite et s'est traduit, notamment, par l'organisation d'une réception monstre à l'occasion du passage d'Édouard, héritier du trône et prince de Galles, en 1860. Malgré le mauvais temps, 20000 personnes se sont pressées dans les rues pour accueillir l'invité royal, chacun tentant de rivaliser d'adresse dans sa profession de foi monarchiste. En 1878, la nomination du marquis de Lorne au poste de gouverneur général du Canada suscita un semblable soulèvement de loyalisme. Un journaliste québécois écrivit :

Nous ne voulons pas médire des autres villes, toutes loyales; mais on doit reconnaître que notre vieille cité, qui a été si longtemps la tête et le cœur du pays, a conservé le don de s'enflammer à la vue des personnages revêtus de l'autorité souveraine, et qu'elle porte dans les manifestations de son enthousiasme un élan qu'on ne retrouve pas dans les démonstrations plus réservées qui se font ailleurs. ([s.a.], $1878: 2$ )

Parmi les facteurs qui expliquent cette différence de ton loyaliste, mentionnons la forte présence anglophone dans la ville. En 1851, plus du tiers de sa population était anglo-saxonne. S'il est vrai que la moitié de la population de Montréal était anglo-saxonne, soit une proportion encore plus élevée, les contacts entre les deux groupes linguistiques étaient différents dans l'une et l'autre agglomérations. Selon Fernand Ouellet, les classes moyennes et celles des petits ouvriers de Québec dépendaient davantage de la classe anglaise que celles de Montréal, car elles étaient moins bien articulées à l'arrière-pays et manifestaient une moins grande solidarité.

«Ainsi Montréal faisait figure de ville plus continentale. [...] En dépit de son infériorité numérique, la population canadienne-française y formait un bloc compact solidaire de la zone rurale, ce qui était beaucoup moins vrai pour Québec. » (F. Ouellet, $1959: 316)$

À cela s'ajoutait le fait que la garnison militaire de Québec regroupait, selon les années, jusqu'à 3000 hommes, et que la ville était dominée par la fine silhouette de la Citadelle. Enfin, la majorité des fonctionnaires qui habitaient la capitale étaient d'origine anglo-saxonne. La présence des soldats dans les rues, la concentration du commerce et de l'industrie entre des mains anglaises, la forte proportion d'anglophones dans l'administration publique donnaient à la capitale « l'allure d'une ville britannique » (F. Ouellet, 1959:314). L'opportunisme de Québec avait une autre origine : on peut avancer qu'il découlait aussi 
du statut de capitale de la ville, statut qui en faisait d'emblée un lieu de pouvoir (J.-M. Lebel, J. Saint-Pierre et Y. Beauregard, 1997) ${ }^{8}$. Après avoir longtemps maintenu cette position enviable, Québec ne fut définitivement minorisé au sein de l'ensemble canadien qu'au moment de la signature de l'Acte de l'Amérique du nord britannique de 1867 et le transfert de la capitale fédérale à Ottawa. Pendant une bonne partie du $\mathrm{XIX}^{\mathrm{e}}$ siècle, la ville s'enorgueillissait de la présence en son sein de personnages de marque. Même si le Canada-Uni, simple colonie anglaise, ne pouvait reconnaître d'ambassadeurs, un véritable corps consulaire fut maintenu à Québec, avec entre autres des envoyés de la France, des ÉtatsUnis, de la Belgique, du Danemark, de la Norvège, du Portugal et de la Prusse (C. Blais, G. Gallichan, F. Lemieux et J. Saint-Pierre, 2008 : 312 et 348). Devenue capitale de la province de Québec, la ville a continué à regrouper, quoiqu'à une plus petite échelle, une foule de ministres, de députés et d'employés du gouvernement. Elle a perdu rapidement de son faste, mais les traditions s'étiolent moins facilement et Québec s'est ainsi pensé comme une ville de prestige bien après la perte de son statut de capitale du Canada-Uni.

Le statut de capitale avait deux conséquences majeures. En premier lieu, Québec était connu au XIX ${ }^{\mathrm{e}}$ siècle pour l'organisation de banquets, des bals, des fêtes et des réceptions très courus par les membres de la magistrature et $\mathrm{du}$ corps consulaire, les officiers de la garnison et les autres dignitaires locaux. On soulignait comment des habitudes de vie luxueuses avaient peu à peu envahi les mœurs ([s.a.], 1843 : 2). Dans une causerie datée de 1867, Hector Fabre parlait de Québec comme d'une ville aristocratique qui se passionnait pour les sorties mondaines.

Ne pas être de la société ! châtiment terrible, peine infamante à laquelle une femme bien née préférera toujours la gêne, le pain sec. (H. Fabre, 1867 : 503)

En deuxième lieu, l'administration publique constituait par la force des choses une des plus importantes activités économiques de la municipalité.

La classe politique et les élites canadiennes de l'ancienne capitale et aussi les milieux d'affaires anglophones québécois [...] profitaient de la proximité du gouverneur et de ses conseillers, [lesquels] étaient aussi les fournisseurs de biens et de services nécessaires à l'administration. (G. Galichan, $2007: 4$ )

Or, ce genre de milieu aurait encouragé une disposition générale à flatter les hommes du pouvoir comme moyen d'avancement plutôt que de se fier à l'esprit d'entreprise individuel (C., 1877 : 302-306). Pendant longtemps, les critiques de

8. De 1608 à 1759, Québec fut le chef-lieu de la Nouvelle-France. Après la Conquête, elle devint, de 1760 à 1791, la capitale de la Province of Quebec, puis de 1791 à 1840, la capitale du Bas-Canada. En février 1840, Kingston est devenu la capitale du Canada-Uni, avant de céder ce statut à Montréal en 1843, mais l'incendie du parlement a provoqué une nouvelle fois le déplacement de la capitale selon un drôle de système d'alternance. C'est ainsi que Toronto accueillit les parlementaires canadiens jusqu'en 1851 et puis de 1855 à 1859, et Québec eut cet honneur de 1851 à 1855 et de 1859 à 1866. 
l'administration coloniale ont d'ailleurs donné à l'élite corrompue qui régnait sur les destinées du Bas-Canada le nom de "Clique du château ", en référence au château Saint-Louis, où résidait le gouverneur et où se trouvaient les bureaux du gouvernement. Pour Louis-Joseph Papineau, député de Montréal et leader du Parti patriote, l'envie de briller dans les somptueux bals du gouverneur général aurait créé une coterie d'intrigants qui se plaisaient à peser sur la marche des événements par des intrigues et des louanges, comme jadis à la cour des rois. Les fêtes organisées par le gouverneur général, les places offertes par le gouvernement, les contrats distribués par l'élite politique, tout cela avait insidieusement créé une dépendance des habitants face au pouvoir impérial. Les courtisans, écrivait Papineau dans une lettre à sa femme, auraient monté les escaliers du Château Saint-Louis « comme la Scala Santa à Rome, où les dévots et les dévotes ont de leurs front et genoux usé la pierre " (Papineau, 2 mars 1826, cité par F. Ouellet, p. 317). Ce jugement était partagé par maints observateurs de Montréal. Encore en 1856, des articles parus dans le Pays dénonçaient comment la «passion des places ", c'est-à-dire la course pour les emplois publics, régnait dans le district de Québec. Les auteurs stigmatisaient «cette obstination de Québec à la curée des places et des profits à faire dans l'exploitation du gouvernement» ([s.a.], 1856a: 3). L'ambition de vivre aux dépens de la collectivité y aurait été hissée au niveau d'un véritable système d'exploitation, la quête des honneurs et des profits étant peu à peu devenue «le but unique de toutes les luttes» ([s.a.], 1856b:2).

La proximité du pouvoir impérial - sous la forme du gouverneur ou du parlement colonial - n'a pas seulement influencé les attitudes des habitants de la capitale, elle a aussi joué un rôle dans le développement de l'Église de Québec. Sous la gouverne de la France, le clergé de la Nouvelle-France s'était plié au césarisme dominant de l'époque ${ }^{9}$, et la Conquête n'avait pas changé cette posture, le gouvernement anglais ne se gênant pas pour intervenir directement dans les affaires de l'Église. La soumission, sinon la docilité du clergé canadien face aux autorités coloniales, justifiée par l'adage bien connu de saint Paul selon lequel il n'y a point d'autorité qui ne vienne de Dieu, était encouragée par la présence du gouverneur à Québec. Inversement, l'installation de Jean-Jacques Lartigue (1777-1840) comme évêque en titre à Montréal en 1836 a pu marquer une étape importante de l'affranchissement de l'Église canadienne, Lartigue se sentant «plus libre de l'influence du gouverneur»(M. Trudel, 1963: 61) du fait que ses affaires étaient plus lointaines ${ }^{10}$. L'hypothèse selon laquelle la proximité avec le pouvoir a pu jouer un rôle dans la tendance plus gallicane de l'Église de Québec est confirmée par une distinction qu'il faut faire, non seulement entre le bas et le haut clergé catholique, mais entre la basse et la haute ville de Québec.

9. Gustave Lanctôt écrivait un peu brutalement : «L'Église canadienne était l'absolue prisonnière de l'État » (1929: 41-43 et 76).

10. Sur la fidélité de l'Église catholique canadienne au pouvoir impérial, lire D. Thério (1998). 
De fait, les commentateurs ont toujours perçu une différence entre les attitudes du haut clergé et du bas clergé au Québec en général, et à Québec en particulier $^{11}$. Le premier a toujours été plus prompt au XIX ${ }^{\mathrm{e}}$ siècle à afficher ses sentiments loyalistes que le second. Cela se conçoit très bien si on s'accorde avec l'idée que la proximité avec le pouvoir encourage une certaine realpolitik catholique (L. Dion, 1975). Les élites supérieures éprouvent non seulement une solidarité de classes, mais sont plus susceptibles de négocier des arrangements sur la base d'une mutualité d'intérêts. Cette pente était accentuée dans le contexte d'une Église raidie par la menace (souvent imaginaire) des mouvements de contestations populaires (d'abord républicains et francs-maçons) et qui pressait ses fidèles de se soumettre tout entier à leurs "princes".

Il est intéressant de noter que l'on peut affirmer sensiblement la même chose de la division entre la basse ville et la haute ville de Québec (L. Noppen, C. Paulette et M. Tremblay, 1979. V. Laflamme, 2001). Dans l'imaginaire des citoyens, mais aussi dans ses réalités sociodémographiques, la capitale était en effet alors coupée en deux. En haute-ville étaient situés le parlement, la cathédrale, le château Saint-Louis, la citadelle, les belles et vastes résidences de la Grande-Allée, et en basse ville les manufactures et les quartiers ouvriers. La haute ville abritait une population généralement plus cossue, plus bourgeoise et plus anglophone, et la basse ville une population plus ouvrière et plus nettement canadiennefrançaise. Dans l'imaginaire populaire, la haute ville a toujours paru suspecte d'excès d'anglophilie, et la basse ville de débordement nationaliste. Par exemple, le Cercle catholique de Québec comptait, parmi ses membres, le curé de SaintRoch, François-Xavier Gosselin, un ultramontain notoire qui s'occupait jusqu'en 1885 de la plus grosse paroisse de la basse ville. La Vérité, l'hebdomadaire de Tardivel, avait également ses bureaux à Saint-Roch.

Encore une fois nous retrouvons la même variation du sentiment royaliste selon que l'on s'approche ou s'éloigne du centre politique. Notons au passage, en conclusion de cette section, que la relation réciproque était aussi vraie : certains observateurs notaient comment le clergé catholique avait une plus grande influence sur les affaires coloniales quand la capitale du Canada-Uni déménageait à Québec. «Le siège du gouvernement fut transporté à Québec, écrivait George Brown, et les ministres furent plus que jamais sous l'influence de la Hiérarchie romaine " (cité par L. Groulx, 1952 : 527). Les rencontres entre les partisans du trône et de l'autel ne favorisaient pas que les premiers, à l'évidence. Par exemple, le grand vicaire du diocèse de Québec de 1850 à 1879, Charles-Félix Cazeau, avait acquis une telle réputation d'être l'éminence grise dans les coulisses du parlement, qu'on lui attribua le surnom de cardinal Antonelli (secrétaire d'État sous Pie IX) du Canada.

11. Cela est vrai dès la fondation de la colonie de la Nouvelle-France (J.-C. Falardeau, $1952: 214-229)$. 


\section{Un gallicanisme " papiste "}

$\mathrm{Au} \mathrm{XIX}^{\mathrm{e}}$ siècle, les ultramontains soutenaient que le pouvoir civil devait toujours céder devant le pouvoir ecclésiastique, car, en cas de doute, le jugement du pouvoir religieux, inspiré ou sinon dicté par le pape, ne pouvait être erroné. L'Église était pourtant de moins en moins en position de donner des instructions à l'État. Au Canada, des politiciens libéraux comme Wilfrid Laurier tout autant que des politiciens conservateurs comme George-Étienne Cartier étaient réputés gallicans. Cela se comprend, puisque les hommes d'État tendent naturellement à accroître les prérogatives du gouvernement et à chercher à limiter ou instrumentaliser celles des autres puissances. L'Église catholique du XIX ${ }^{\mathrm{e}}$ siècle, qui proférait une doctrine intransigeante, a donc souvent dû fuir les confrontations et accepter des compromis avec le pouvoir séculier qui lui permettaient de négocier des arrangements " tactiques » satisfaisants. On le devine à voir les envoyés pontificaux que le Vatican déléguait au Canada : le prélat choisi comme visiteur apostolique par Rome au Canada en 1877 était un Irlandais, George Conroy, et celui envoyé en octobre 1883 était un Belge, Dom Henri Smeulders. En mars 1897, ce fut un marquis né de père espagnol et de mère anglaise, Mgr Merry del Val, qui descendit à Québec comme délégué apostolique. Tous se firent connaître pour leurs positions accommodantes vis-à-vis des milieux anglophones et protestants.

S'il est vrai que le clergé n'était pas insensible à l'influence de l'État, il demeure que la réalité nationale canadienne a fait en sorte de moduler les rapports entre Église catholique et gouvernements de manière pour le moins originale. Le fait que le pays englobait des communautés anglophone et francophone ainsi que des groupes catholique et protestant ne pouvait laisser l'Église canadienne indifférente, et en particulier l'Église de Québec qui, parce qu'elle se trouvait plus près du pouvoir impérial, était davantage amenée à composer avec des milieux politiques naturellement hostiles. Il semblait donc plus réaliste et plus stratégique de prôner des doctrines de concorde et de compromis. Un catholique libéral le disait :

Dans un pays où l'autorité souveraine est protestante et dont la population la plus nombreuse professe un culte différent du nôtre, l'intérêt et le simple bon sens, à part les considérations toutes puissantes de foi et de dogme, nous montrent et nous font sauter aux yeux l'absurdité de doctrines menant droit aux églises nationales et aux schismes. ([s.a.], 1870)

Taschereau était le premier à reconnaître que l'idéal d'harmonie entre la cité terrestre et la cité céleste était chose difficile à atteindre et qu'il fallait, contrairement à ce que souhaitaient les ultramontains "ultramontés ", user de prudence dans ce domaine. Soucieux de préserver la bonne entente entre l'élément de langue française et celui de langue anglaise, le Saint-Siège était persuadé que l'approche tempérée de Taschereau était la plus sage et la plus susceptible de porter fruit. 
Il tenait tout particulièrement à ne pas entremêler trop fortement religion, nation et politique, puisque le projet de conversion de l'Amérique du nord demandait de ne pas se compromettre avec les nationalistes canadiens-français. Cette prudence diplomatique ne fit que s'accentuer avec l'élection du pape du Ralliement, Léon XIII, qui prenait en 1878 le relais du pape du Syllabus. De toute façon, la romanité a toujours été plus complexe et plus flexible dans les faits que ce que les ultras, dans leur rêve de reconquista, voulaient bien imaginer. À cet égard, le comportement plus agressif de Bourget ou de Laflèche, qui souhaitaient édifier sur les rives du Saint-Laurent une société française et catholique « assez robuste pour résister à la culture anglo-protestante dominante »(R. Perin, 2008 : 44), semblait lourd de complications pour l'Église.

Rome jugeait que, par ses idées et par ses actions, Bourget manquait de sensibilité à l'égard de la réalité anglo-protestante qui entourait les Canadiens français en tant que peuple. Cet évêque, à leurs yeux trop attaché à son groupe ethnique, mettait en cause les bons rapports qu'entretenait l'Église avec les autorités civiles du Canada et de la Grande-Bretagne. En même temps, il menaçait le rêve que caressait le Saint-Siège de convertir le monde anglo-saxon. (R. Perin, 2008 : 218)

Les préfets de la Propagande croyaient que la mixité religieuse du pays exigeait plus de retenue. L'un d'eux écrivait en 1881 :

Il faudra exhorter les évêques à observer par rapports aux affaires politiques la plus grande réserve, eu égard particulièrement au danger qu'il y a de provoquer à une guerre violente contre l'Église les protestants déjà inquiets et irrités contre le clergé sous prétexte d'ingérence indue dans les élections politiques. (Lettre du cardinal Jean Siméoni à Mgr Alexandre Taschereau, Rome, 13 septembre 1881, publiée dans [s.a.], $1881: 494)$

Le Witness de Montréal se réjouissait des positions modérées adoptées par le Saint-Siège :

Le Pontife actuel a fait preuve de libéralité et de prudence dans chacun de ses actes jusqu'ici. L'Archevêque de Québec vient de rendre publiques deux lettres de Rome dans lesquelles les évêques ultramontains du Canada, qui ont même dépassé Pie IX dans son temps, reçoivent la meilleure rebuffade qu'ils n'aient jamais reçue de leur vie. On leur dit qu'ils ne doivent pas se mêler de politique; et l'une des raisons invoquées, c'est que les protestants sont irrités par l'influence qu'ils exercent [...] (cité par J.-P. Tardivel, 1881, 4)

Les ultramontains, qui pensaient bien pouvoir compter sur l'appui indéfectible du Saint-Siège, se retrouvaient dans la drôle de position de devoir contester les décisions du Vatican. Par exemple, quand Narcisse-Eutrope Dionne, rédacteur du Courrier du Canada, avait refusé de publier une protestation contre ceux qui accusaient Taschereau d'avoir trompé les congrégations romaines, ce dernier avait déclaré : "Si les ultramontains ne se tiennent pas tranquilles, je ferai venir de Rome des foudres qui écraseront tous ces tapageurs » (lettre de Dionne à Mgr Laflèche, cité par R. Rumilly, 1938 : 173). 
Le Canada français offrait l'étonnant paradoxe d'ultramontains en rébellion contre le Saint-Siège et de gallicans qui ne cessaient d'en appeler à la soumission aux décrets romains. Comble de l'ironie, on vit même des ultramontains s'adresser aux tribunaux ou aux parlements pour contester des décisions de Rome! Ce ne fut que lorsque la lutte parut définitivement perdue, avec entre autres l'élection du Parti libéral à Ottawa en 1896, que Tardivel admit l'inévitable et s'emporta dans La Vérité contre les conservateurs qui s'obstinaient à contester les décisions papales : "On dit : le Pape est vieux, le Pape est loin, et surtout le Pape a été abominablement renseigné par son délégué [...]. C’est du gallicanisme [...], de ce gallicanisme importé de France dès la fondation de la colonie, et qui n'a jamais été complètement extirpé du sol canadien " ${ }^{12}$ (cité par R. Rumilly, 1938 : 418). C'est ainsi que les ultramontains canadiens-français finirent par se plier à des décisions du Vatican qui entérinaient les politiques d'un Parti libéral fédéral honni.

\section{La perte du statut de capitale}

Le statut de capitale de Québec n'a pas été sans infléchir jusqu'à un certain point les relations entre l'évêché et le pouvoir civil. Le fait que l'Église doive transiger avec un pouvoir anglais et protestant qui entendait la respecter pour autant qu'elle le servait dans son entreprise de domination impériale a eu pour effet de la rendre plus circonspecte dans ses ambitions prosélytes. Forcée de côtoyer de près le gouverneur général et des administrateurs anglais, elle a reconnu, davantage qu'à Trois-Rivières ou Montréal, la nécessité d'adopter des attitudes souples et conciliantes. Ses appels répétés à se soumettre à l'autorité d'une reine qui était en même temps chef d'une Église protestante ne manquent pas de piquant, mais, minorité tolérée dans un empire britannique encore habité par des sentiments antipapistes, elle savait que la paix et la liberté religieuses constituaient la meilleure garantie de son développement dans la colonie.

L'Église du Canada occupe aujourd'hui une belle position; nous sommes de ceux qui s'imaginent qu'elle n'a pu arriver là que par une tactique sage, et qui veulent la continuation de cette tactique. Nous désirons que les traditions de l'épiscopat sur ce point ne cessent jamais d'être notre règle de conduite à tous [...] ([s.a.], $1870: 2$ )

En se situant au cœur de la colonie, l'archevêché de Québec était mieux à même de saisir les périls d'un catholicisme trop intégral. Le contexte urbain est cependant bouleversé en 1867 , lorsque Québec perd pour de bon son statut de capitale du Canada-Uni. La primauté de l'archevêché de Québec au sein de l'ensemble catholique canadien est elle aussi brisée, lors du concile provincial de

12. Cette déclaration de Tardivel fut faite à l'occasion des débats entourant l'encyclique Affari Vos. Adressée à l'épiscopat canadien en décembre 1897, cette encyclique devait régler la question de l'enseignement du français et de la religion dans les écoles du Manitoba, un enjeu qui concernait l'avenir du groupe francophone dans tout le Canada. 
1868, avec le redécoupage de la carte ecclésiastique canadienne et la subséquente division de la province ecclésiastique de Québec qui s'étendait autrefois vers l'ouest. Désormais, le clergé catholique québécois n'a plus à porter la responsabilité de l'unité nationale canadienne et, coupé du reste du pays, il s'offre comme relativement homogène du point de vue de la langue et des traditions, en dépit de la présence d'une importante minorité irlandaise anglophone.

Bien que les habitudes diplomatiques et aristocratiques restent longtemps gravées dans les opinions, sinon dans les mœurs du clergé, peu à peu, la culture religieuse sera affectée par les changements politiques et socioéconomiques. En déclin économique dans la deuxième moitié du XIX ${ }^{e}$ siècle (N. Lanouette, 2008) et en processus de francisation accélérée, Québec incarne désormais, en tant que capitale provinciale, le foyer historique du peuple canadien-français, et non plus le centre du Dominion britannique. Alors qu'il était plus facile autrefois pour l'évêque de Québec, qui conversait régulièrement avec les gouverneurs généraux et les politiciens des deux Canadas, de se situer dans une perspective globale, la provincialisation de la ville de Québec fait en sorte que les successeurs sur le siège épiscopal de Québec percevaient davantage les choses à travers le prisme des intérêts de leur nationalité. Cette évolution urbaine contribua à modifier graduellement la sensibilité des catholiques québécois dans un sens plus conservateur et plus nationaliste, alors que Montréal, au même moment, devenait au contraire toujours plus cosmopolite sous la gouverne de l'évêque Paul-Napoléon Bruchési (1897-1939) (F. Primeau, 2007).

Manifestation de cette évolution, Louis-Nazaire Bégin, l'évêque de Québec (1898-1925) autrefois réputé très libéral, accusait Rome, à la fin du XIX ${ }^{\mathrm{e}}$ siècle, de faire le jeu du Parti libéral fédéral. Il jugeait ce parti coupable « de toutes les fourberies et de toutes les trahisons " parce qu'il n'avait pas suffisamment affirmé les droits nationaux des Canadiens français dans leur lutte contre les visées assimilatrices des anglophones. Il lui semblait normal à cet égard que l’Église du Québec contribue à la défense de la nationalité canadienne-française.

Notre peuple de langue française est prêt à subir et à souffrir toutes les persécutions, ouvertes ou déguisées, plutôt que d'abandonner des droits qu'il considère comme sacrés [...] Et nous, ses chefs spirituels [...] nous nous exposerions à perdre sa confiance, si nous ne le soutenons pas [...] dans ses luttes et ses revendications. (Bégin à Benoît XV, 13 avril 1915, cité par D. Tremblay, 1995 : 9)

Le problème, c'est que Bégin voyait le pouvoir sur les destinées de l'Église au Canada lui échapper de plus en plus, l'épiscopat canadien-français étant lentement écarté de la gouvernance ecclésiastique nationale par les membres du clergé anglophone, et d'abord irlandais, qui occupaient une majorité des sièges épiscopaux au pays. La nomination de Mgr Donato Sbarretti au poste de délégué apostolique au Canada par le Saint-Siège de 1902 à 1910 n'avait fait que jeter de l'huile sur le feu. 
La langue anglaise, [écrivait le délégué en 1909], est inévitablement destinée à dominer de plus en plus ce pays. Plus vite on reconnaîtra une telle situation, mieux ce sera pour tous. (Lettre de Mgr Sbarretti au cardinal Rafael Merry Del Val, 25 mai 1909, cité par D. Tremblay, 1995 : 11)

À ses yeux, les tentatives de maintien de la langue française n'avaient pas "d'autres effets que ceux d'endommager les vrais intérêts de l'Église » (ibid.). Mgr Sbarretti aurait confié à Henri Bourassa, le grand leader nationaliste du tournant du $\mathrm{Xx}^{\mathrm{e}}$ siècle, qu'il valait mieux pour eux, en définitive, que les Canadiens de langue française apprennent l'anglais, comme les immigrants allemands ou italiens (Henri Bourassa à Stagni, 6 août 1913, cité dans ibid. : 13). Ajoutée à la marginalisation politique, la marginalisation en quelque sorte ecclésiastique dans l'ensemble canadien va accentuer le repli de plus en plus manifeste du clergé de Québec sur les frontières de la nationalité canadienne-française.

L'un des apports de cette étude historique de l'impact du statut de capitale sur les destinées du clergé québécois, est sans doute d'avoir mieux fait ressortir les clivages qui existaient au XIX ${ }^{\mathrm{e}}$ siècle entre les principales agglomérations de la province. Sans exagérer les fractures qui divisent à cette époque l'Église catholique québécoise, il n'en demeure pas moins que cette institution est moins homogène que ne le laisse croire la nécessité pour elle de parler d'une seule voix. Marqués par des réalités urbaines différentes (homogénéité ethnique, présence d'une forte minorité anglophone ou protestante, cercles libéraux plus ou moins actifs, etc.), les évêchés ne sont pas insensibles au milieu dans lequel ils gravitent au quotidien. De là les oppositions qui ont défrayé la chronique dans la deuxième moitié du XIX ${ }^{\mathrm{e}}$ siècle et ont dressé entre autres Montréal et Québec l'une contre l'autre.

Ces tensions découlaient en partie de guerres de clochers, de rivalités urbaines et d'intérêts locaux antagonistes, mais on ne saurait mésestimer complètement la modification que la position de Québec comme capitale de la colonie britannique apportait au regard du clergé catholique. Ce texte a tenté d'identifier certains éléments qui permettent d'illustrer le biais introduit par des relations plus intenses entre le pouvoir religieux et le pouvoir civil dans le contexte bien particulier du Canada-Uni. La coupure entre gallicans et ultramontains et entre libéraux et conservateurs s'est trouvée à recouper en partie l'opposition entre la capitale et la métropole. Que la ville de Québec ait été elle-même animée par des courants contrastés qui suivaient mutatis mutandis, au moins dans la conscience populaire, les lignes tracées entre le haut et le bas clergé et la haute et la basse ville, ne fait que confirmer la thèse générale : il semble bien que la réalité du pouvoir politique ne soit pas sans inviter à l'adoption de stratégies ecclésiales différentes. 
Même au plus fort de son intransigeantisme, Rome ne l'a-t-elle pas reconnu en appuyant au Canada des politiques libérales qu'elle semblait vouer aux gémonies partout ailleurs ?

Jean-Philippe WARREN

CRIDAQ (Centre de recherche interdisciplinaire sur la diversité au Québec)

Université du Québec à Montréal

Concordia University Research Chair for the Study of Quebec

jphwarren@sympatico.ca

\section{Bibliographie}

[s.a.], 1843, "[The selection of the future...] ", Montreal Gazette, 30 septembre, p. 2.

[s.a.], 1856a, "Le district de Montréal et celui de Québec ", Le Pays, 9 avril, p. 3.

[s.a.], 1856b, " Justice pour tous - justice pour nous ", Le Pays, 2 avril, p. 2-3.

[s.a.], 1870, "Ultramontains et gallicans ", L'Opinion publique, 12 février, p. 1-2.

[s.a.], 1878, "Le marquis de Lorne ", L’Événement, 6 décembre, p. 2.

[s.a.], 1881a, "Laval et ses partisans à Montréal », Le Monde, 17 octobre, p. 4.

[s.a.], 1881b, « L'affaire de l'Université-Laval », L'opinion publique, 20 octobre, p. 494.

BERNARD Jean-Paul, 1971, Les Rouges; libéralisme, nationalisme et anticléricalisme an milieu du XIX ${ }^{e}$ siècle, Montréal, Presses de l’Univ. du Québec.

Blais Christian, Gallichan Gilles, Lemieux Frédéric et Saint-Pierre Jocelyn, 2008, Québec. Quatre siècles d'une capitale, Québec, Les Publications du Québec.

C. P., 1877, "Québec ancien et moderne (suite et fin) ", Le Foyer domestique, III-5, p. 302-306.

Duclos de Celles Alfred, 1881, "Montréal et Québec », L’Opinion publique, 22 septembre, p. 416.

Desjardins Paul, 1944, Le collège Sainte-Marie de Montréal, Montréal, Imp. du Messager.

Dion Léon, 1975, Nationalisme et politique au Québec, Montréal, Hurbubise HMH.

Dupus Jean-Claude, 2006, « Mgr Elzéar-Alexandre Taschereau et le catholicisme libéral au Canada français (1850-1898) ", thèse en histoire, Québec, Univ. Laval.

FABRe Hector, 1867, "Causerie sur Québec ", Revue canadienne, 4, 7 juillet, p. 501-509.

Falardeau Jean-Charles, 1952, "Rôle et importance de l'Église au Canada français ", Esprit, 193-194, p. 214-229.

FERGUSON Louis B., 2009, "Histoire de la gouvernance du Canada français : influence et injonction entre les pouvoirs ecclésiastiques et civils ", La Revue des Sciences de Gestion, 239-240, p. 127-147.

GaLICHAN Gilles, 2007, "La ville de Québec et le défi de la capitale (1841-1865) », Les Cahiers des dix, 61, p. 1-41.

Gagnon Marcel-A., 1965, Le ciel et l'enfer d'Arthur Buies, Presses de l'Univ. Laval, Québec.

GIRARD Mathieu, 1967, «La pensée politique de Jules-Paul Tardivel », Revue d'histoire de l'Amérique française, 21-3, p. 397-428.

GreER Allen et Robichaud Léon, 1989, « La rébellion de 1837-1838 au Bas-Canada : une approche géographique ", Cahiers de Géographie du Québec, XXXIII-90, p. 345-377. 
Groulx Lionel, 1952, "Le choix de la capitale au Canada ", Revue d'histoire de l'Amérique française, 5-4, p. 521-530.

Hayward Annette, 1991, "La rivalité Québec-Montréal au début du siècle ", Voix et images, 16-3, p. 514-524.

LAFLAMme Valérie, 2001, "Familles et modes de résidence en milieu urbain québécois au début du Xx siècle : l'exemple de la ville de Québec, 1901 ", Cahiers québécois de démographie, 30-2, p. 261-288.

Lamonde Yvan, 2000, Histoire sociale des idées, 1760-1896, Montréal, Fides.

Lanctôt Gustave, 1929, L'Administration de la Nouvelle-France, Paris, Honoré Champion.

LANOUETTE Nicolas, 2008, "Québec comme laboratoire urbain ", Cahiers de géographie du Québec, 52-145, p. 43-61.

LaVallée André, 1974, Québec contre Montréal. La querelle universitaire 1876-1891, Montréal, Les Presses de l’Univ. de Montréal.

Lebel Jean-Marie, Saint-Pierre Jacques et Beauregard Yves, 1997, La capitale. Lieu $d u$ pouvoir, Sainte-Foy, Commission de la Capitale Nationale et Les Publications du Québec.

Luigi [Pelletier Alexis], 1873, Il y a du libéralisme et du gallicanisme en Canada, Montréal, Imp. Le Franc-Parleur.

Noppen Luc, Paulette Claude et Tremblay Michel, 1979, Québec, trois siècles d'architecture, Québec, Libre expression.

Ouellet Fernand, 1959, "Papineau et la rivalité Québec-Montréal (1820-1840) », Revue d'histoire de l'Amérique française, 13-3, p. 311-327.

PERIN Roberto, 1993, Rome et le Canada. La bureaucratie vaticane et la question nationale, 1870-1903, Montréal, Boréal.

-, 2008, Ignace de Montréal. Artisan d'une identité nationale, Montréal, Boréal, 2008.

Primeau Francis, 2007, «Le libéralisme dans la pensée religieuse de Mgr Bruchési », Mens, 7-2, printemps, p. 241-277.

RHEAULT Marcel J., 2011, La rivalité universitaire Québec-Montréal revisitée 150 ans plus tard, Québec, Septentrion.

Roy Pierre-Georges, 1997 [1901], La famille Taschereau, Lévis, Imprimerie Mercantile, p. 7-8.

Rudin Ronald, 1997, Faire de l'histoire au Québec au vingtième siècle, Sillery, Septentrion.

Rumilly Robert, 1938, Mgr Laflèche et son temps, Montréal, Les Éditions du Zodiaque, 1938.

-, 1962, "Mgr Laflèche et les ultramontains ", Revue d'histoire de l'Amérique française, 16-1, p. 95-101.

SAINT-PIERRE Télesphore, 1894, Histoire du commerce canadien-français de Montréal, 1535-1893. Un souvenir, Montréal, Sabiston.

SAVArd Pierre, 1967, "Le Cercle catholique de Québec, 1876-1897 », Culture, XXVIII-1, p. 3-17.

Sylvain Philippe et VoIsIne Nive, 1991, Histoire du catholicisme québécois, tome II, Réveil et consolidation, 1840-1898, Montréal, Boréal.

Tardivel Jules-Paul, 1881, À son Éminence le Cardinal J. Simeoni, Préfet de la Sacrée Congrégation de la Propagande, Québec, [s.n.].

THÉRIO Adrien, 1998, Un siècle de collusion entre le clergé et le gouvernement britannique : anthologie des mandements des évêques, 1760-1867, Montréal, XYZ. 
Tremblay Donald, 1995, "Mgr Pellegrino Francesco Stagni, o.s.m., et l’Église canadienne, 1910-1918 ", thèse de doctorat, Univ. Laval.

Trudel Marcel, 1963, "La servitude de l'Église catholique du Canada français sous le régime anglais ", rapports annuels de la Société historique du Canada, 42-1, p. 42-64.

Villeneuve Paul Y., 1981, "La ville de Québec comme lieu de continuité ", Cahiers de géographie du Québec, 25-64, p. 49-60.

\section{La ville de Québec comme foyer " libéral " au xix ${ }^{\mathrm{e}}$ siècle Les catholiques canadiens-français entre opportunisme et ultramontanisme}

Dans la deuxième moitié du XIXe siècle, l'antagonisme entre Québec et Montréal s'est très souvent manifesté lors de polémiques religieuses. Or, ce n'est pas Montréal qui incarnait à cette époque, aux yeux de maints observateurs, le haut lieu de la pensée catholique progressiste, mais plutôt Québec. La question dès lors se pose : comment se fait-il que le catholicisme canadien-français a semblé se montrer plus ouvert dans une ville que dans l'autre? Pour comprendre ce fait, cet article s'attarde sur les dynamiques sociopolitiques propres à la capitale québécoise, dynamiques liées à la plus ou moins grande proximité des élites religieuses avec le pouvoir civil. En revenant sur ce facteur, une première lunette d'approche s'offre pour saisir la spécificité de Québec comme capitale d'une colonie, puis d'une province.

Mots-clés : Québec, Montréal, Église catholique, capital politique, ultramontanisme.

\section{The city of Quebec as a "liberal" center in the $19^{\text {th }}$ century French Canadian Catholics between opportunism and ultramontanism}

In the second half of the $19^{\text {th }}$ century, the antagonism between Quebec and Montreal often manifested itself around religious polemics. Interestingly, at that time, Montreal did not represent, in the eyes of many observers, the centre of Catholic progressive thought, but Quebec did. The question therefore arises: how come French Canadian Catholicism seemed more open in one city than in the other? To understand this opposition, this article analyses the social dynamics that characterised the city of Quebec, dynamics that were linked to the religious elites' greater proximity to political power. By focusing on this factor, this paper offers a different lens to define Quebec's specificity as the capital of a colony, before it became the capital of a province.

Key words: Quebec, Montreal, Catholic Church, political capital, ultramontanism.

\section{La ciudad de Quebec como foco "liberal" en el siglo XIX Los católicos franco-canadienses entre oportunismo y ultramontanismo}

En la segunda mitad del siglo XIX, el antagonismo entre Quebec y Montreal se manifestó a menudo a través de polémicas religiosas. A los ojos de numerosos observadores, no era Montreal la que encarnaba en esta época el lugar principal del pensamiento católico progresista, sino más bien Quebec. Se plantea entonces una pregunta: ¿cómo es posible que el catolicismo franco-canadiense se haya mostrado aparentemente más abierto en una ciudad que en otra? Para comprender este hecho, 
244 - ArChives de SCIENCES SOCIALES DES RELIGIONS

este artículo se detiene en las dinámicas sociopolíticas propias de la capital de Quebec, dinámicas ligadas a la proximidad más o menos estrecha de las élites religiosas con el poder civil. Volviendo sobre este factor, se propone un primer enfoque para comprender la especificidad del Quebec como capital de una colonia primero, luego de una provincia.

Palabras clave: Quebec, Montreal, Iglesia Católica, capital política, ultramontanismo. 